\title{
Rett syndrome: Novel correlations linking $>96 \%$ genotype, disease severity, and seizures
}

\author{
Lourdes M. Rodriguez ${ }^{\mathrm{a}, 1}$, Alan K. Percy ${ }^{\mathrm{b}, 1}$ and Gary R. Cutter ${ }^{\mathrm{c}, 1, *}$ \\ ${ }^{a}$ Civitan International Research Center, University of Alabama at Birmingham, Birmingham, \\ University of Chicago to Lourdes, USA \\ ${ }^{\mathrm{b}}$ Department of Pediatrics, Civitan International Research Center, University of Alabama \\ at Birmingham, Birmingham, AL, USA \\ ${ }^{\mathrm{c}}$ Department of Biostatistics, University of Alabama at Birmingham School of Public Health, \\ University of Alabama at Birmingham, Birmingham, AL, USA
}

\begin{abstract}
.
BACKGROUND: Rett Syndrome (RTT), an incurable neurodevelopmental disorder associated in $>96 \%$ with the X-linked gene, MECP2 includes seizures, among its most difficult issues, impacting many features and increasing morbidity and mortality. Linking these seizures with clinical severity in RTT is critical for estimating risk and guiding therapy.

OBJECTIVE: Our primary purpose was to identify associations between type and frequency of seizures, disease severity, and specific $M E C P 2$ mutations to address the hypothesis that seizure frequency correlates with specific mutations and directly impacts clinical severity.

METHODS: Mutation, seizure type and frequency, and clinical severity assessed by the Clinical Severity Scale (CSS) were extracted from the 5211 Natural History Study of Rett Syndrome and Related Disorders [1]. This involved observations from 222 Persons with classic or variant RTT and $M E C P 2$ mutation positive non-Rett diagnoses. Descriptive analyses were assessed utilizing SPSS software. Mutations include $R 106 \mathrm{~W}, R 133 C, R 168 X, R 294 X, R 306 C$, other point mutations, and early truncations.

RESULTS: Greater frequency of generalized seizures and seizures of any type were associated with $R 106 \mathrm{~W}$ mutations; $R 168 X$ mutations had the highest disease severity, and $R 133 C$ mutations had the lowest disease severity.

CONCLUSION: Important correlations exist across several common $M E C P 2$ mutations, including the novel association between generalized seizure frequency and mild CSS.
\end{abstract}

Keywords: Rett syndrome, seizures, mutations, clinical severity, MECP2

\section{Introduction}

Rett Syndrome (RTT) is a rare neurodevelopmental disorder affecting more than one in ten thousand girls. RTT, due to mutations in the X-linked gene, $M E C P 2$, is based on specific clinical criteria including partial or complete loss of spoken language and hand use, stereotypic movements, and abnormal or absent gait (Neul et al, 2010) [2]. Clinical severity varies according to the MECP2 genetic mutation,

\footnotetext{
${ }^{1}$ All authors contributed equally to the manuscript.

${ }^{*}$ Corresponding author: Gary R. Cutter, RPHB 414, 1665 University Boulevard, Birmingham, AL 35294, USA. Tel.: +1 205975 5048/ 205975 2540; E-mail: cutterg@uab.edu.
} 
X-chromosome inactivation (unpublished observations), potentially other genes, and environmental influences including specific therapies and/or medications $[3,4]$. Seizures in RTT may be subtle and not readily identified or may be generalized. Generalized and other seizures, among the many difficult features of RTT, are common; their occurrence may increase morbidity and mortality, as they may lead to aspiration pneumonias in those with already impaired swallowing abilities [5].

Further study is needed to identify which of those affected with RTT have the highest risk of seizures by type and frequency and the highest risk of greater clinical severity. Identifying these populations is key to implementing prophylactic intervention and pharmacological treatment.

The purpose of this study is to identify and determine associations between type and frequency of seizures, clinical severity and specific $M E C P 2$ mutations to address the hypothesis that seizure frequency is correlated with specific mutations and directly impacts clinical severity.

\section{Methods}

\subsection{Standard protocol approvals, registrations, and patient consents}

\subsubsection{Human study approval}

Human study approval was obtained from the Institutional Review Boards of each participating site prior to study participation. The parent of each participant provided informed consent for study participation prior to study enrollment. The IRB waived the assent of each participant because of her/his cognitive impairment. A Certificate of Confidentiality was provided by NICHD.

\subsubsection{Clinical trials gov}

Two non-interventional clinical trials protocols (NCT00299312 and NCT02738281) [1, 6] were involved during this more than 16-year study.

\subsection{Data source}

Data from Persons with Rett Syndrome (PwRS) were obtained from the five-year observational study, 5211 Natural History Study of Rett Syndrome and Related Disorders [1]. Using PwRS IDs in lieu of Personal Identifiers, all relevant information was compiled and securely maintained. Data were collected from 222 PwRS with common MECP2 mutations, which included at least one observation per PwRS. PwRS have either clinical features of classic RTT $(193 / 222=86.9 \%)$, variant RTT $(20 / 222=9.0 \%)$, or a MECP2 mutation positive non-Rett diagnosis $(9 / 222=4.1 \%)$; however, the majority of our sample have a RTT diagnosis $(n=213,213 / 222=96.0 \%)$.

\subsection{Data extraction procedures}

Data collection included assessing the $M E C P 2$ genotype and other factors influencing seizure frequency, such as medications, epileptic chronic diagnoses, and clinical severity. This project compares the mutations $R 106 W, R 133 C, R 168 X, R 294 X, R 306 C$, other point mutations, and early truncations to the frequency and severity of the seizures.

Additional data for seizures and spells influencing seizure frequency were recorded: the average and maximum duration of seizures; seizure type; and the number of spell symptoms from those named and listed in the 5211 Natural History Study [1] seizure database. The data used from the 5211 Natural History Study [1] database were physician-recorded data from individual visits of PwRS throughout the enrollment period of the study. In total, this study dataset is comprised of eighteen independent and outcome variables, shown in Appendix 2. 
Chronic epilepsy diagnoses and RTT diagnoses were also recorded to account for its potential contribution to PwRS clinical severity of seizures at a given visit, which can influence the overall clinical severity score, as it is the sum of severity ratings for each of the following thirteen criteria of the CSS:

Age of Onset Regression, Onset of Stereotypes, Head Growth*, Somatic growth*, Independent sitting*, Ambulation by Exam*, Hand Use, Scoliosis, Language by Exam*, Nonverbal Communication*, Respiratory Dysfunction*, Autonomic symptoms*, Epilepsy*

*=specific to each visit

CSS ranges from 0 to 58, based on 7 items with ordinal scores $0-4$ and 6 items with ordinal scores $0-5$. These clinical severity scores reflect the sum of severity ratings of each symptom, or the collective disease severity; disease severity and seizure severity at each visit (observation) per PwRS were recorded in data collection.

\subsection{Data analysis}

Using SPSS software, descriptive statistics were utilized to summarize the data. The frequency of seizures was compared with clinical severity and specific $M E C P 2$ mutations using chisquare tests. The frequency of mutations and seizure occurrence, and the first seizure of each type in categories (multiple per day, daily, weekly, more than weekly, weekly-monthly, less than monthly) per PwRS by mutation were summarized by percentages and means.

The mean severities were compared amongst the mutation groupings using analysis of variance with adjustments for multiple comparisons by Tukey's Highest Significant Difference (HSD) approach. Additionally, descriptive summary statistics were computed for frequencies of clinical disease severity, frequency of seizure over time, average seizure duration, and seizure severity were computed to enable comparisons, such as distributions of frequency of seizures in two ranges of time (less than a week, and one week or more) by mutation per PwRS, of average seizure duration by mutation per PwRS, and isolated distributions of severity of each of the six types of seizures by mutation per PwRS.

To assess the outcome variables described and genotype while controlling for seizure type, we included its occurrence testing the "ever" presence of a seizure type recorded in the dataset for each PwRS. "Absent" or missing data for seizure type were not included in the calculation of the rates of clinical severity of any seizure type and no imputation was attempted.

\subsection{Data availability}

Anonymized data not provided in this article are available upon request from Dr. Alan Percy for purposes of replicating procedures and results.

\section{Results}

\subsection{PwRS descriptors}

Each individual has a disease-causing $M E C P 2$ genotype. The most frequent $M E C P 2$ mutations in our sample (Table 1) were in $R 168 X$ (61 individuals $-27.5 \%$ ), $R 133 C$ (50 individuals $-22.5 \%$ ), and $R 294 X$ (40 individuals $-18.0 \%$ ); less frequent genotypes in our sample were in $R 306 C$ (24 individuals $-10.8 \%), R 106 \mathrm{~W}$ (23 individuals $-10.3 \%$ ), other point mutations (19 individuals $-8.6 \%$ ), and early truncations (5 individuals $-2.4 \%$ ). 
Table 1

Summarized data

\begin{tabular}{|c|c|c|}
\hline$M E C P 2$ Mutation & $\mathrm{N}$ & $\%$ of Total \\
\hline Early Truncations & 5 & $2.3 \%$ \\
\hline Other Point Mutations & 19 & $8.6 \%$ \\
\hline$R 106 W$ & 23 & $10.4 \%$ \\
\hline$R 133 C$ & 50 & $22.5 \%$ \\
\hline$R 168 X$ & 61 & $27.5 \%$ \\
\hline$R 294 X$ & 40 & $18.0 \%$ \\
\hline$R 306 C$ & 24 & $10.8 \%$ \\
\hline \multicolumn{3}{|l|}{ Seizure Type } \\
\hline Absence Seizure & 1 & $0.4 \%$ \\
\hline Atonic Seizure & 6 & $2.7 \%$ \\
\hline Complex Partial Seizure & 33 & $14.9 \%$ \\
\hline Generalized Seizure & 65 & $29.3 \%$ \\
\hline Other Seizure & 2 & $0.9 \%$ \\
\hline Rett Spell & 34 & $15.3 \%$ \\
\hline Absent & 81 & $36.5 \%$ \\
\hline \multicolumn{3}{|l|}{ Average Spell Duration } \\
\hline$<1$ Minute & 95 & $47.0 \%$ \\
\hline $1-<5$ Minutes & 45 & $22.3 \%$ \\
\hline$>10$ Minutes & 11 & $5.5 \%$ \\
\hline Absent & 51 & $25.2 \%$ \\
\hline \multicolumn{3}{|l|}{ Seizure Frequency } \\
\hline Less than Weekly & 167 & $75.2 \%$ \\
\hline >Weekly & 16 & $7.2 \%$ \\
\hline Weekly - Monthly & 19 & $8.5 \%$ \\
\hline$<$ Monthly & 47 & $21.2 \%$ \\
\hline Absent & 85 & $38.3 \%$ \\
\hline Weekly or More & 55 & $24.8 \%$ \\
\hline Multiple per Day & 14 & $6.3 \%$ \\
\hline Daily & 15 & $6.8 \%$ \\
\hline Weekly & 26 & $11.7 \%$ \\
\hline
\end{tabular}

The other point mutations in our study include p.Asp156Glu (D156E) $(n=1)$, p.Leu100Val (L100V) (1), p.Pro101Arg (P101R) (2), P152R (9), p.Pro225Arg (P225R) (4), p.Pro322Leu (P322L) (2). Mutations in R133C include p.Arg133Cys (R133C) (46) and p.Ser134Cys (S134C) (4). Mutations in R106W include $p . \operatorname{Arg} 106 \operatorname{Trp}(R 106 \mathrm{~W})(21)$ and $p . \operatorname{Arg} 106 \operatorname{Gln}(R 106 Q)(2)$.

Of the 20 PwRS with atypical or variant Rett syndrome, 2 individuals had Atypical Congenital (Rolando) Variant Rett Syndrome, 3 individuals had Atypical Preserved Speech (Zapella) Variant Rett syndrome, and 15 individuals had Atypical/Variant unspecified Rett syndrome.

\subsection{Clinical severity associations}

The CSS ranged from 0 to 42 . The PwRS clinical severity scores were assessed using One way Anova calculating the mean $\pm \mathrm{SEM}$ of $28.6 \pm 1.0$ for $R 168 X, 20.3 \pm 1.2$ for $R 294 X, 20.3 \pm 1.5$ for $R 306 C$, $17.3 \pm 1.1$ for $R 133 C, 20.8 \pm 1.7$ for other point mutations, and $25.6 \pm 3.3$ for early truncations. The overall test of severity amongst these groupings were significantly different with a $p$-value of $<0.0001$. Using Tukey's HSD multiple comparisons testing procedure, we identified 6 of the 21 pairwise com- 
Table 2

Tukey Kramer's honest significant difference connecting letters report for disease severity across the seven MECP2 mutation groups (CSS)

\begin{tabular}{lllll}
\hline Mutation type & & & & Mean \\
\hline$R 168 X$ & $\mathrm{~A}$ & & & 28.7 \\
$R 106 W$ & $\mathrm{~A}$ & $\mathrm{~B}$ & & 25.9 \\
Early Truncations & $\mathrm{A}$ & $\mathrm{B}$ & $\mathrm{C}$ & 25.6 \\
Other Point Mutations & & $\mathrm{B}$ & $\mathrm{C}$ & 20.8 \\
$R 306 C$ & & $\mathrm{~B}$ & $\mathrm{C}$ & 20.3 \\
$R 294 X$ & & $\mathrm{~B}$ & $\mathrm{C}$ & 20.3 \\
$R 133 C$ & & $\mathrm{C}$ & 17.3 \\
\hline
\end{tabular}

Levels not connected by the same letter are significantly different.

parisons to be significantly different: $R 168 X$ was significantly different from other point mutations $(p=0.0019) R 306 C(p=0.0002), R 294 X(p<0.0001)$ and $R 133 C(<0.0001) . R 133 C$ was significantly different from $R 168 X$ and $R 106 \mathrm{~W}(p=0.0002)$ and the others were not significantly different from each other (Table 2).

\subsection{Seizure frequency}

\subsubsection{Total sample}

Of the 141 PwRS in our sample with seizure frequency data (65\%), the proportion of PwRS who experienced seizure frequency less often than weekly, was $56.7 \%(80 / 141)$; exceeding that for seizure frequencies of weekly or more, $43.3 \%$ (61/141).

There was little difference in the frequent episodes of seizures by mutations, for seizures $<$ weekly vs. $\geq$ weekly. The results were $R 168 X 73.8 \%<1$ per week $(n=45$ and $16 ;<1$ week, $\geq 1$ week respectively), $R 133 C 80.0 \%(40,10), R 294 X 72.5 \%(29,11), R 306 C 70.8 \%(17,7), R 106 W 65.2 \%(15,8)$, other point mutations $84.2 \%(16,3)$, and early truncations $100 \%(5,0)$.

\subsubsection{Seizure type}

The most frequent type of seizure observed in this study was a generalized seizure (at least one occurrence in 72 PwRS - 32.4\%); followed by complex partial seizures ( $\geq 1$ in 38 PwRS - 17.1\%); and Rett spells ( $\geq 1$ in 27 PwRS - 12.2\%); the least common type of seizure observed were atonic seizures ( $\geq 1$ in 7 PwRS $-3.2 \%)$, absence seizures ( $\geq 1$ in 2 PwRS $-0.9 \%)$, and other episodes $(\geq 1$ in 2 PwRS -. 0.9\%).

See Appendix 3 for seizure type classifications.

The average rate per PwRS for each type of seizure by phenotypes were: atonic seizures $\left(\frac{19 \text { observed }}{7 \text { patients }} \approx 2.7\right.$ occurrences per patient $)$, Rett spells $\left(\frac{65 \text { observed }}{27 \text { patients }} \approx 2.4\right.$ occurrences per patient $)$, complex partial seizures $\left(\frac{73 \text { observed }}{38 \text { patients }} \approx 1.9\right.$ occurrences per patient $)$, and generalized seizures $\left(\frac{127 \text { observed }}{72 \text { patients }} \approx 1.8\right.$ occurrences per patient $)$.

The occurrence of generalized seizures among the seven MECP 2 mutation types are shown in Table 3 with no significant differences by mutation $\left(\chi^{2} \approx 5.5, d f=6, a=.05, p=.49\right)$. Given the small number of first occurrences of complex partial seizures, Rett spells, atonic seizures, absence seizures, and other episodes, we also are not able to detect significant differences among $M E C P 2$ genotypes for these seizure types. Although the frequency of generalized seizures is lowest in the other point 
Table 3

Distribution of generalized seizures across 7 MECP2 genotypes

\begin{tabular}{lcccccccc}
\hline Ever Generalized & & \multicolumn{7}{c}{ MECP2 Mutation } \\
\cline { 3 - 9 } $\begin{array}{l}\text { Frequency } \\
\text { Column \% }\end{array}$ & $\begin{array}{c}\text { Other Point } \\
\text { Mutations }\end{array}$ & $R 106 W$ & $R 133 C$ & $R 168 X$ & $R 294 X$ & $R 306 C$ & $\begin{array}{c}\text { Early } \\
\text { Truncations }\end{array}$ & Total \\
\hline No & 14 & 11 & 28 & 33 & 26 & 11 & 3 & 126 \\
& 73.7 & 47.8 & 56.0 & 54.1 & 65.0 & 45.8 & 60.0 & \\
Yes & 5 & 12 & 22 & 28 & 14 & 13 & 2 & 96 \\
26.3 & 26.3 & 52.2 & 44.0 & 45.9 & 35.0 & 54.2 & 40.0 & 222 \\
Total & 19 & 23 & 50 & 61 & 40 & 24 & 5 & 24 \\
\hline
\end{tabular}

mutations, $26.3 \%$, compared to others, which range from $35.0 \%$ to $54.2 \%$, generalized is the one statistically significant controlled seizure type that differs $(p=0.019)$ in the comparisons of seizure type duration and severity.

The most frequent episodes of generalized seizures $(<1$ week, $\geq 1$ week) by point mutation were $R 168 X 66.7 \%<1$ week $(22,11), R 133 C 64.0 \%(16,9), R 294 X 87.5 \%(28,4), R 306 C 86.7 \%(13,2)$, and $R 106 \mathrm{~W} 61.5 \%(8,5)$; the frequency of episodes of seizures for other point mutations was $85.7 \%$ and early truncations $100 \%$ were $(6,1)$ and $(2,0)$, respectively.

The distribution of the seizure frequency for complex partial, absence, atonic, and other seizures alongside Rett spells were too sparse to calculate statistical significance. However, after $R 168 X$, mutations, $R 133 C$ was associated with a higher frequency of generalized seizures (occurring at least once in 22 PwRS with an average 1.8 occurrences per PwRS), although not statistically different from the other mutations combined $(p=0.31)$.

\subsection{Seizure duration}

In total, 95 PwRS (42.7\%) had at least one seizure with a duration of less than one minute; 56 PwRS (25.2\%) have had at least one occurrence of a seizure lasting one minute or longer.

Excluding $71 \mathrm{PwRS}$ without seizure duration data, the percent with spell duration in time $(<1$ minute, $\geq 1$ minute) by mutations were $R 168 X 66.0 \%(31,16), R 133 C 61.5 \%(16,10), R 294 X 63.0 \%(17,10)$, $R 306 C 68.8 \%(11,5)$, and $R 106 \mathrm{~W} 55.6 \%(10,8)$; the frequency of episodes for other point mutations and early truncations were $57.1 \%(8,6)$ and $66.7 \%(2,1)$, respectively.

\subsection{Seizure severity}

The severity of the first generalized seizure for each PwRS in the study is displayed in Table 4, and is recorded for the 96 PwRS with at least one generalized seizure occurrence. 24\% (23/96) had seizures with a severity deemed zero and $19.8 \%$ (19/96) had seizures rated as 5 on the severity scale.

The mean (standard deviation) severity of generalized seizures for MECP2 mutations in $R 168 X$, $R 133 C, R 294 X, R 306 C, R 106 \mathrm{~W}$, other point mutations, and early truncations are $2.4 \pm 2.0,2.0 \pm$ $2.0,2.1 \pm 1.8,1.7 \pm 1.9,2.6 \pm 1.6,2.8 \pm 1.8$, and $2.0 \pm 0$, respectively. The average generalized seizure severity is 2.22 .

Overall, no differences were found in generalized seizure severity and most were graded on average below the CSS mid-value of 2.5. Averages above the mid-value were found in other point mutations and Rl06W. 
Table 4

Clinical severity of generalized seizures per PwRS for 7 MECP2 genotypes

\begin{tabular}{lcccc}
\hline $\begin{array}{l}\text { Generalized } \\
\text { Severity }\end{array}$ & Frequency & Percentage & $\begin{array}{c}\text { Cumulative } \\
\text { Frequency }\end{array}$ & $\begin{array}{c}\text { Cumulative } \\
\text { Percentage }\end{array}$ \\
\hline 0 & 23 & 24.0 & 23 & 24.0 \\
1 & 21 & 21.9 & 44 & 45.9 \\
2 & 11 & 11.4 & 55 & 57.3 \\
3 & 14 & 14.6 & 69 & 71.9 \\
4 & 8 & 8.3 & 77 & 80.2 \\
5 & 19 & 19.8 & 96 & 100.0 \\
\hline
\end{tabular}

\section{Discussion}

Review of the literature indicates that Classic Rett Syndrome populations with Common MECP2 point mutations p.Arg168X (R168X), p.Arg106Trp (R106W), p.Arg255X (R255X), and p.Arg270X $(R 270 X)$ have higher clinical severity scores, while point mutations in p.Arg133Cys (R133C), p.Arg294X (R294X), and p.Arg306Cys (R306C) present with milder disease severity [3, 7-10]. R168X has presented with larger frequency of seizures in longitudinal study of classic RTT [5].

Mutations in $R 168 X$ had the highest clinical severity mean of $28.7 \pm 7.2$, and mutations in $R 133 C$ had the lowest mean of $17.3 \pm 7.9$. These statistically different results coincide within the mean CSS and standard deviations of Typical RTT (25.7 \pm 6.7 for $R 168 X$ and $18.0 \pm 6.2$ for $R 133 C$ ) and Atypical RTT (31.0 \pm 3.6 for $R 168 X$ and $13.4 \pm 5.7$ for $R 133 C$ ) in previous research [3].

In our entire sample, the most common frequency of seizures was less than one episode per week. $R 133 C$ has the highest percentage of PwRS with less than one seizure episode per week, notwithstanding its relatively high percent with generalized seizures occurring weekly or more. $R 306 C, R 168 X$ (respectively) and $R 106 \mathrm{~W}$ present the highest frequency of seizures and generalized seizures occurring weekly or more. No significant differences in generalized seizure severity were identified.

In this study, we found that individuals with mutations in $R 106 \mathrm{~W}$ experience a higher frequency of seizures with at least one generalized seizure and one seizure of any type weekly or more often than weekly. The consistency of these results with investigations of seizure onset $[11,12]$, in conjunction with the higher percent of epilepsy in $R 106 \mathrm{~W}$ in previous research [13], highlight the association between $R 106 \mathrm{~W}$ and seizure occurrence. Our research expands the investigation of seizures in the $R 106 W$ population $[5,9,14]$, showing that $R 168 X$ and $R 133 C$ follow after $R 106 W$ in having the highest percentages of generalized seizure occurrence. Extended research of the expanding populations of individuals with mutations in $R 106 \mathrm{~W}$ would increase our understanding this association since these patients were treated in various manners. More therapeutic studies might enable implementation of prophylactic interventions in the RTT populations most at risk.

Antiepileptic medications administered to PwRS presents a limitation and potential source of confounding. Since there are a large number of medications and dosages and compliance operative yielding varied effectiveness in regulating the occurrence of seizures in each PwRS, we were unable to separate whether the associations found are breakthrough disease under treatment or mutation related. Treatment by indication is thus confounded with the results in this study as with other clinical observational studies. Nevertheless, unless treatment works better in one mutation versus another, our results point out groups at risk. Another limitation of this study is the random presence of X-chromosome inactivation in RTT PwRS that causes uncontrolled expression of MECP2 genotypes. Additionally, RTT is a genetically sporadic disease carried on the $\mathrm{X}$ chromosome and while one of the largest studies of 
RTT, the limitations of sample size and enrollment of PwRS in a natural history cohort impacts this and almost all rare disease clinical research.

Despite these limitations, this study provides a strong foundation for planning future trials addressing seizure prevention and treatment that can improve RTT morbidity and mortality.

\section{Conclusions}

We were able to identify correlations that further confirm previous results in RTT clinical research literature $[3,7,8,13]$. Alongside studying seizure frequency, we compared seizure duration among these mutation types and located differences among the mutation groups, although no statistically significant differences were found in the average generalized seizure severity among the mutations studied.

The analyses revealed that $R 133 C$, a common $M E C P 2$ point mutation is associated with a milder phenotype, presenting with a higher frequency of generalized seizures. This follows after the highest generalized seizure frequency of $R 168 X$ ( 28 individuals with $\geq 1$ occurrence), a common $M E C P 2$ point mutation associated with a more severe phenotype. Whether these effects are caused by the mutation or treatment is unknown.

Our study may help to inform predictability from diagnosis and neurological impairments, prophylactic intervention, and pharmacological treatment. The strength of this study is based in the careful data collection from the 5211 Natural History Study [1], the largest sample size of directly evaluated individuals with this very rare condition.

\section{Conflict of interest}

The authors have no conflict of interest, competing interest, or representation of any institution.

\section{Acknowledgments}

The authors recognize the important contributions of all site investigators in capturing the relevant data utilized in this study. These include Mustafa Sahin and David Lieberman at Boston Children's Hospital (Harvard), Eric Marsh at Children's Hospital of Philadelphia (University of Pennsylvania), Sarika Peters and Jeffrey Neul at Vanderbilt University, Steven Skinner at Greenwood Genetic Center, Daniel Glaze at Baylor College of Medicine, Peter Heydemann at Rush Medical Center, Arthur Beisang and Timothy Feyma at Gillette Children's Hospital, Shannon Standridge at Cincinnati Children's Hospital, Robin Ryther at Washington University (St. Louis), Timothy Benke at University of ColoradoDenver, Richard Haas at University of California San Diego, and Mary Jones at University of San Francisco-Oakland Children's Hospital.

\section{Author Disclosures}

Lourdes Rodriguez: No disclosures.

Alan K. Percy: Dr. Percy is PI of the US Natural History Study funded by NICHD: HD061222. He participates in clinical trials with Anavex, Acadia Pharmaceuticals, GW Pharmaceuticals, and in investigator-initiated trial with Ketamine, all for Rett syndrome. He is also a consultant to Anavex and Acadia. He has no conflicts with respect to the Natural History Study. 
Gary R. Cutter:

Data and Safety Monitoring Boards: Astra-Zeneca, Avexis Pharmaceuticals, Biolinerx, Brainstorm Cell Therapeutics, Bristol Meyers Squibb/Celgene, CSL Behring, Galmed Pharmaceuticals, Horizon Pharmaceuticals, Hisun Pharmaceuticals, Mapi Pharmaceuticals LTD, Merck, Merck/Pfizer, Opko Biologics, OncoImmune, Neurim, Novartis, Ophazyme, Sanofi-Aventis, Reata Pharmaceuticals, Teva pharmaceuticals, VielaBio Inc, Vivus, NHLBI (Protocol Review Committee), NICHD (OPRU oversight committee).

Consulting or Advisory Boards: Biodelivery Sciences International, Biogen, Click Therapeutics, Genzyme, Genentech, GW Pharmaceuticals, Klein-Buendel Incorporated, Medimmune, Medday, Neurogenesis LTD, Novartis, Osmotica Pharmaceuticals, Perception Neurosciences, Recursion/Cerexis Pharmaceuticals, Roche, TG Therapeutics.

Dr. Cutter is employed by the University of Alabama at Birmingham and President of Pythagoras, Inc. a private consulting company located in Birmingham AL.

\section{References}

[1] A.K. Percy, 5211 Natural History Study of Rett Syndrome and Related Disorders [Internet] NCT02738281. Birmingham (AL): University of Alabama at Birmingham. 2014 - [cited 2020 Jul 22]. Available from: https://clinicaltrials.gov/ct2/show/record/NCT02738281.

[2] J.L. Neul, W.E. Kaufmann, D.G. Glaze, et al., Rett syndrome: revised diagnostic criteria and nomenclature, Ann Neurol 68(6) (2010), 944-950. doi:10.1002/ana.22124

[3] V.A. Cuddapah, R.B. Pillai, K.V. Shekar, et al., Methyl-CpG-binding protein 2 (MECP2) mutation type is associated with disease severity in Rett syndrome, J Med Genet 51(3) (2014), 152-158. doi:10.1136/jmedgenet-2013-102113

[4] J.L. Neul, P. Fang, J. Barrish, et al., Specific mutations in methyl-CpG-binding protein 2 confer different severity in Rett syndrome, Neurology 70(16) (2008), 1313-1321. doi:10.1212/01.wnl.0000291011.54508.aa

[5] D.C. Tarquinio, W. Hou, A. Berg, et al., Longitudinal course of epilepsy in Rett syndrome and related disorders, Brain 140(2) (2017), 306-318. doi:10.1093/brain/aww302

[6] A.K. Percy, 5201 Rett Syndrome Natural History: Genetic and Physical Characteristics of Rett Syndrome [Internet] NCT00299312. Birmingham (AL): University of Alabama at Birmingham. 2006 - [cited 2020 Aug 4]. Available from: https://clinicaltrials.gov/ct2/show/record/NCT00299312.

[7] R.E. Amir and H.Y. Zoghbi, Rett syndrome: methyl-CpG-binding protein 2 mutations and phenotype-genotype correlations, Am J Med Genet 97(2) (2000), 147-152. doi:10.1002/1096-8628(200022)97:2<147::aid-ajmg6>3.0.co;2-o.

[8] H. Archer, J. Evans, H. Leonard, et al., Correlation between clinical severity in patients with Rett syndrome with a p.R168X or p.T158M MECP2 mutation, and the direction and degree of skewing of X-chromosome inactivation, $J$ Med Genet 44(2) (2007), 148-152. doi:10.1136/jmg.2006.045260

[9] T. Charman, T. Neilson, V. Mash, et al., Dimensional phenotypic analysis and functional categorisation of mutations reveal novel genotype-phenotype associations in Rett syndrome, Eur J Hum Genet 13 (2005), 1121-1130. https://doi.org/10.1038/sj.ejhg.5201471.

[10] A. Nissenkorn, et al., Epilepsy in Rett syndrome-Lessons from the Rett networked database, Epilepsia 56, 569-576. doi:10.1111/epi.12941

[11] L. Jian, L. Nagarajan, N. de Klerk, et al., Predictors of seizure onset in Rett syndrome, The Journal of Pediatrics 149(4), 542-547.e3. doi: https://doi.org/10.1016/j.jpeds.2006.06.015

[12] L. Jian, L. Nagarajan, N. de Klerk, D. Ravine, J. Christodoulou and H. Leonard, Seizures in Rett syndrome: an overview from a one-year calendar study, Eur J Paediatr Neurol 11(5) (2007), 310-317. doi:10.1016/j.ejpn.2007.02.008

[13] D.G. Glaze, A.K. Percy, S. Skinner, et al., Epilepsy and the natural history of Rett syndrome, Neurology 74(11) (2010), 909-912. doi:10.1212/WNL.0b013e3181d6b852

[14] M. Pintaudi, M.G. Calevo, A. Vignoli, et al., Epilepsy in Rett syndrome: clinical and genetic features, Epilepsy Behav 19(3) (2010), 296-300. doi:10.1016/j.yebeh.2010.06.051 


\section{Appendix 1}

Authors

\begin{tabular}{llc}
\hline Name & Location & Contribution \\
\hline Lourdes M. Rodriguez, HSD & $\begin{array}{c}\text { University of Alabama at } \\
\text { Birmingham, } \\
\text { Birmingham }\end{array}$ & $\begin{array}{c}\text { Major role in the design and conceptualized } \\
\text { study; major role in the acquisition of the data; } \\
\text { interpreted the data; drafted and revised the } \\
\text { manuscript for intellectual content. }\end{array}$ \\
Alan K. Percy, MD & $\begin{array}{c}\text { University of Alabama at } \\
\text { Birmingham, }\end{array}$ & $\begin{array}{c}\text { Design and conceptualized the study; major role } \\
\text { in acquisition of the data; major role in revision }\end{array}$ \\
& $\begin{array}{l}\text { Birmingham } \\
\text { oniversity of Alabama at manuscript for intellectual content. } \\
\text { Biry R. Cutter, PhD }\end{array}$ & $\begin{array}{c}\text { Major role in analysis and interpretation of the } \\
\text { data; major role in the revision of the }\end{array}$ \\
& Birmingham & manuscript for intellectual content.
\end{tabular}

\section{Appendix 2}

Eighteen outcome variables from data collection

1) PwRS Identification Number

2) Gender

3) Rett Syndrome Diagnosis

4) Visit (i.e.: Baseline, Year 1, Year 2...)

5) Average Seizure Frequency (*)

6) Maximum Seizure Frequency (*)

7) Average Duration of Spell

8) Maximum Duration of Spell

9) Type of Seizure

10) Chronic Epilepsy Diagnosis

11) Developmental and Health History Form (Seizures)

12) Seizure type specifics, as per a note by the physician

13) Number of spell features counted as listed in the Natural History Study [1] Seizure Database. (**)

14) Antiepileptic Medications

15) Seizure Rating at visit (Clinical Severity Scale)

16) Clinical Severity Score at visit (Clinical Severity Scale)

17) $M E C P 2$ genotypes collapsible into three levels of specificity: Grouping 1 (gene), Grouping 2 (genetic mutation), and Grouping 3 (type of genetic mutation)

(*) Counts were obtained in clinical longitudinal assessments over the course of the five-year Natural History Study [1]. This seizure frequency data from each visit for which seizure type data were complete were abstracted and analyzed in our research.

(**) Spell features included in the total count:

Abnormal posture, Appears confused, Arms and legs move, Arms or legs stiff, Becomes limp, Breath holding, Eye blinking, Eyes jerk to one side, Eyes roll back in, Falls down, Follows people with eyes, Head jerks to one side, Lips turn blue, Makes guttural noise, Non-rhythmic chant, Occurs in stages, Other specify, Responds to name, Staring, Unresponsive to calling, Other features of spell. 


\title{
Appendix 3
}

Seizure Type Classifications

Seizure types extracted from the 5211 Natural History Study [1] are categorized into six major groups according to the following classification:

\author{
Absence Seizure \\ Atonic Seizure \\ Drop atonic seizures and atonic seizures

\section{Complex Partial Seizure} \\ Complex partial seizure episodes, complex partial, and partial seizures
}

\section{Generalized Seizure}

Generalized, generalized tonic-clonic, generalized grand mal, tonic-clonic seizures

\section{Other Seizures}

Myoclonic seizures, infantile spasms, drop seizures, "seizure" or unspecified seizure

\section{Rett Spell}

